\title{
CERTAIN RESULTS ON KENMOTSU PSEUDO-METRIC MANIFOLDS
}

\author{
DEVARAJA MALLESHA NAIK, VENKATESHA, AND D.G. PRAKASHA
}

Received 21 March, 2019

\begin{abstract}
In this paper, a systematic study of Kenmotsu pseudo-metric manifolds are introduced. After studying the properties of this manifolds, we provide necessary and sufficient condition for Kenmotsu pseudo-metric manifold to have constant $\varphi$-sectional curvature, and prove the structure theorem for $\xi$-conformally flat and $\varphi$-conformally flat Kenmotsu pseudo-metric manifolds. Next, we consider Ricci solitons on this manifolds. In particular, we prove that an $\eta$-Einstein Kenmotsu pseudo-metric manifold of dimension higher than 3 admitting a Ricci soliton is Einstein, and a Kenmotsu pseudo-metric 3-manifold admitting a Ricci soliton is of constant curvature $-\varepsilon$.
\end{abstract}

2010 Mathematics Subject Classification: 53C15; 53C25; 53D10

Keywords: almost contact pseudo-metric manifold, Kenmotsu pseudo-metric manifold, $\varphi$-sectional curvature, conformal curvature tensor

\section{INTRODUCTION}

The study of contact metric manifolds with associated pseudo-Riemannian metrics were first started by Takahashi [10] in 1969. Since then, such structures were studied by several authors mainly focusing on the special case of Sasakian manifolds. The case of contact Lorentzian structures $(\eta, g)$, where $\eta$ is a contact one-form and $g$ is a Lorentzian metric associated to it, has a particular relevance for physics and was considered in [4] and [1]. A systematic study of almost contact semi-Riemannian manifolds was undertaken by Calvaruso and Perrone [3] in 2010, introducing all the technical apparatus which is needed for further investigations.

On the other hand, in 1972 Kenmotsu [9] investigated a class of contact metric manifolds satisfying some special conditions, and after onwards such manifolds are came to known as Kenmotsu manifolds. Recently, Wang and Liu [11] investigated almost Kenmotsu manifolds with associated pseudo-Riemannian metric. These are called almost Kenmotsu pseudo-metric manifolds. In this paper, we undertake the systematic study of Kenmotsu pseudo-metric manifolds.

The first author (D.M.N.) is grateful to University Grants Commission, New Delhi (Ref. No.:20/12/2015(ii)EU-V) for financial support in the form of Junior Research Fellowship. 
The present paper is organized as follows: In Section 2, we give the basics of Kenmotsu pseudo-metric manifold $(M, g)$. Certain properties of Kenmotsu pseudometric manifolds are provided in Section 3. We devote Section 4 to the study of curvature properties of Kenmotsu pseudo-metric manifold $(M, g)$ and gave necessary and sufficient condition for $(M, g)$ to have constant $\varphi$-sectional curvature. In Section 5, we prove necessary and sufficient condition for Kenmotsu pseudo-metric manifold to be $\xi$-conformally flat (and $\varphi$-conformally flat). The last section is focused on the study of Kenmotsu pseudo-metric manifolds whose metric is a Ricci soliton. We show that if $(M, g)$ is a Kenmotsu pseudo-metric manifold admitting a Ricci soliton, then the soliton constant $\lambda=2 n \varepsilon$, where $\varepsilon= \pm 1$. Moreover, we show that if $M$ is an $\eta$-Einstein manifold of dimension higher than 3 admitting Ricci soliton, then $M$ is Einstein. Further we show that, a Kenmotsu pseudo-metric manifold $(M, g)$ of dimension 3 admitting Ricci soliton is of constant curvature $-\varepsilon$, where $\varepsilon= \pm 1$. Finally, an illustrative example is constructed which verifies our results.

\section{PRELIMINARIES}

Let $M$ be a $(2 n+1)$ dimensional smooth manifold. We say that $M$ has an almost contact structure if there is a tensor field $\varphi$ of type $(1,1)$, a vector field $\xi$ (called the characteristic vector field or Reeb vector field), and a 1-form $\eta$ such that

$$
\varphi^{2}=-I+\eta \otimes \xi, \quad \eta(\xi)=1, \quad \varphi \xi=0, \quad \eta \circ \varphi=0 .
$$

If $M$ with $(\varphi, \xi, \eta)$-structure is endowed with a pseudo-Riemannian metric $g$ such that

$$
g(\varphi X, \varphi Y)=g(X, Y)-\varepsilon \eta(X) \eta(Y),
$$

where $\varepsilon= \pm 1$, for all $X, Y \in T M$, then $M$ is called an almost contact pseudo-metric manifold. The relation (2.2) is equivalent to

$$
\eta(X)=\varepsilon g(X, \xi) \text { along with } g(\varphi X, Y)=-g(X, \varphi Y) .
$$

In particular, in an almost contact pseudo-metric manifold, it follows that $g(\xi, \xi)=\varepsilon$ and so, the characteristic vector field $\xi$ is a unit vector field, which is either space-like or time-like, but cannot be light-like.

The fundamental 2-form of an almost contact pseudo-metric manifold is defined by

$$
\Phi(X, Y)=g(X, \varphi Y),
$$

which satisfies $\eta \wedge \Phi^{n} \neq 0$. An almost contact pseudo-metric manifold is said to be a contact pseudo-metric manifold if $d \eta=\Phi$. The Riemannian curvature tensor $R$ is given by $R(X, Y)=\left[\nabla_{X}, \nabla_{Y}\right]-\nabla_{[X, Y]}$, which is opposite to the one used in [3]. The Ricci operator $Q$ is determined by

$$
\operatorname{Ric}(X, Y)=g(Q X, Y),
$$


where Ric denotes the Ricci tensor. In an almost contact pseudo-metric manifold there always exists a special kind of local pseudo-orthonormal basis $\left\{e_{i}, \varphi e_{i}, \xi\right\}_{i=1}^{n}$, called a local pseudo $\varphi$-basis.

Consider the manifold $M \times \mathbb{R}$, where $M$ is an almost contact pseudo-metric manifold. Denoting the vector field on $M \times \mathbb{R}$ by $\left(X, f \frac{d}{d t}\right)$, where $X \in T M, t \in \mathbb{R}$, and $f$ is a smooth function $M \times \mathbb{R}$, we define the structure $J$ on $M \times \mathbb{R}$ by

$$
J\left(X, f \frac{d}{d t}\right)=\left(\varphi X-f \xi, \eta(X) \frac{d}{d t}\right),
$$

which defines an almost complex structure. If $J$ is integrable, we say that the almost contact pseudo-metric structure $(\varphi, \xi, \eta)$ is normal. Necessary and sufficient condition for integrability of $J$ is [3]

$$
[\varphi, \varphi](X, Y)+2 d \eta(X, Y) \xi=0 .
$$

The following can be easily obtained.

Proposition 1. An almost contact pseudo-metric manifold is normal if and only if

$$
\left(\nabla_{\varphi X} \varphi\right) Y-\varphi\left(\nabla_{X} \varphi\right) Y+\left(\nabla_{X} \eta\right)(Y) \xi=0,
$$

where $\nabla$ is the Levi-Civita connection.

An almost Kenmotsu pseudo-metric manifold is an almost contact pseudo-metric manifold with $d \eta=0$ and $d \Phi=2 \eta \wedge \Phi$. A normal almost Kenmotsu pseudo-metric manifold is called a Kenmotsu pseudo-metric manifold [11]. Equivalently, from (2.4) we have the following:

Definition 1. Almost contact pseudo-metric manifold is said to be Kenmotsu pseudometric manifold if

$$
\left(\nabla_{X} \varphi\right) Y=-\eta(Y) \varphi X-\varepsilon g(X, \varphi Y) \xi
$$

From (2.5), we see

$$
\nabla \xi=I-\eta \otimes \xi
$$

A straight forward calculation gives the following:

Proposition 2. On Kenmotsu pseudo-metric manifold $(M, g)$, we have

$$
\begin{gathered}
\left(\nabla_{X} \eta\right) Y=\varepsilon g(X, Y)-\eta(X) \eta(Y), \\
£_{\xi} g=2 g-\varepsilon \eta \otimes \eta, \\
£_{\xi} \varphi=0, \\
£_{\xi} \eta=0,
\end{gathered}
$$

where $£$ denotes the Lie derivative. 


\section{SOME PROPERTIES OF KENMOTSU PSEUDO METRIC MANIFOLDS}

For $X \in \operatorname{Ker} \eta$, either space-like or time-like, the $\xi$-sectional curvature $K(\xi, X)$ and $\varphi$-sectional curvature $K(X, \varphi X)$ are defined respectively as

$$
\begin{aligned}
K(\xi, X) & =\frac{g(R(\xi, X) X, \xi)}{\varepsilon g(X, X)}, \\
K(X, \varphi X) & =\frac{g(R(\varphi X, X) X, \varphi X)}{g(X, X)^{2}} .
\end{aligned}
$$

Now we prove:

Proposition 3. If $(M, g)$ is a Kenmotsu pseudo-metric manifold, then we have

$$
\begin{aligned}
R(X, Y) \xi & =\eta(X) Y-\eta(Y) X, \\
\eta(R(X, Y) Z) & =\eta(Y) g(X, Z)-\eta(X) g(Y, Z), \\
R(X, \xi) Y & =\varepsilon g(X, Y) \xi-\eta(Y) X, \\
R i c(X, \xi) & =-2 n \eta(X) \quad(\Rightarrow Q \xi=-2 n \varepsilon \xi), \\
K(\xi, \cdot) & =-\varepsilon, \\
\left(\nabla_{Z} R\right)(X, Y, \xi) & =\varepsilon\{g(X, Z) Y-g(Y, Z) X\}-R(X, Y) Z .
\end{aligned}
$$

Proof. Equations (2.6) and (2.7) give (3.1). Equations (3.2), (3.3), (3.4) and (3.5) are consequences of (3.1). Equation (3.6) follows from (2.6), (2.7) and (3.1).

Definition 2. An almost contact pseudo-metric manifold for which

$$
\varphi^{2}\left(\nabla_{W} R\right)(X, Y, Z)=0,
$$

for all $X, Y, Z, W \in T M$ is said to be globally $\varphi$-symmetric.

Using (3.2) and (3.6), we have the following:

Corollary 1. A globally $\varphi$-symmetric Kenmotsu pseudo-metric manifold is of constant curvature $-\varepsilon$.

A Kenmotsu pseudo-metric manifold $M$ is said to be $\eta$-Einstein if the Ricci tensor satisfies

$$
\operatorname{Ric}(X, Y)=a g(X, Y)+b \eta(X) \eta(Y),
$$

where $a$ and $b$ are certain smooth functions on $M$. If $b=0$, then $M$ is called an Einstein manifold.

From (3.4), we have

$$
\varepsilon a+b=-2 n .
$$

Contracting (3.7) and using (3.8), we get

$$
a=\left(\frac{r}{2 n}+\varepsilon\right), \quad b=-\left(\frac{\varepsilon r}{2 n}+2 n+1\right),
$$

where $r$ is the scalar curvature. Thus, we have: 
Proposition 4. A Kenmotsu pseudo-metric manifold $(M, g)$ is $\eta$-Einstein if and only if

$$
\operatorname{Ric}(X, Y)=\left(\frac{r}{2 n}+\varepsilon\right) g(X, Y)-\left(\frac{\varepsilon r}{2 n}+2 n+1\right) \eta(X) \eta(Y) .
$$

In particular, we have the following:

Corollary 2. A Kenmotsu pseudo-metric manifold $(M, g)$ is Einstein if and only if

$$
\operatorname{Ric}(X, Y)=-2 n \varepsilon g(X, Y) \text {. }
$$

Proposition 5. If the Kenmotsu pseudo-metric manifold $(M, g)$ is $\eta$-Einstein, then

$$
X(b)+2 b \eta(X)=0,
$$

for $n>1$, and for any vector field $X \in T M$.

Proof. Equation (3.10) is equivalent to

$$
Q Y=a Y+b \varepsilon \eta(Y) \xi,
$$

where $a$ and $b$ are as in (3.9). It is well known that

$$
\operatorname{div} Q=\frac{1}{2} D r,
$$

where $D$ denotes the gradient. Equations (3.13) and (3.14) yields to

$$
(n-1) Y(a)=\varepsilon\{\xi(b) \eta(Y)+2 n b \eta(Y)\} .
$$

For $Y=\xi$, it gives $\xi(b)=-2 b$, and so we get (3.12) for $n>1$.

Corollary 3. If $b$ (or $a)$ is constant in an $\eta$-Einstein Kenmotsu pseudo-metric manifold, then it is Einstein.

\section{Curvature PROPERTIES OF KENMOTSU PSEUdo METRIC MANIFOLDS}

First we prove the following Lemma which is very useful in subsequent sections.

Lemma 1. On Kenmotsu pseudo-metric manifold $(M, g)$, we have the following identities:

$$
\begin{aligned}
R(X, Y) \varphi Z-\varphi R(X, Y) Z= & \varepsilon\{g(Y, Z) \varphi X-g(X, Z) \varphi Y \\
& +g(X, \varphi Z) Y-g(Y, \varphi Z) X\}, \\
R(\varphi X, \varphi Y) Z=R(X, Y) Z+ & \varepsilon\{g(Y, Z) X-g(X, Z) Y \\
& +g(Y, \varphi Z) \varphi X-g(X, \varphi Z) \varphi Y\} .
\end{aligned}
$$

Proof. The Ricci identity shows that

$$
\nabla_{X} \nabla_{Y} \varphi-\nabla_{Y} \nabla_{X} \varphi-\nabla_{[X, Y]} \varphi=R(X, Y) \varphi-\varphi R(X, Y) .
$$

Computing the left-hand side using (2.5) yields (4.1). The equation (4.2) is a result of (4.1). 
Note that the necessary and sufficient condition for a Sasakian pseudo-metric manifold to have constant $\varphi$-sectional curvature $c$ is [10]

$$
\begin{aligned}
4 R(X, Y) Z= & (c+3 \varepsilon)\{g(Y, Z) X-g(X, Z) Y\} \\
& +(\varepsilon c-1)\{\eta(X) \eta(Z) Y-\eta(Y) \eta(Z) X\} \\
& +(c-\varepsilon)\{\eta(Y) g(X, Z) \xi-\eta(X) g(Y, Z) \xi+g(X, \varphi Z) \varphi Y \\
& -g(Y, \varphi Z) \varphi X+2 g(X, \varphi Y) \varphi Z\} .
\end{aligned}
$$

Here we prove:

Theorem 1. The necessary and sufficient condition for a Kenmotsu pseudo-metric manifold $M$ to have constant $\varphi$-sectional curvature $c$ is

$$
\begin{aligned}
4 R(X, Y) Z= & (c-3 \varepsilon)\{g(Y, Z) X-g(X, Z) Y\} \\
& +(c+\varepsilon)\{\varepsilon \eta(X) \eta(Z) Y-\varepsilon \eta(Y) \eta(Z) X \\
& +\eta(Y) g(X, Z) \xi-\eta(X) g(Y, Z) \xi+g(X, \varphi Z) \varphi Y \\
& -g(Y, \varphi Z) \varphi X+2 g(X, \varphi Y) \varphi Z\} .
\end{aligned}
$$

Proof. Suppose that $M$ has constant $\varphi$-sectional curvature $c$. Then for all vector fields $U, V \in \operatorname{Ker} \eta$, we have

$$
R(U, \varphi U, U, \varphi U)=-\operatorname{cg}(U, U)^{2} .
$$

Using (4.1), we get

$$
\begin{aligned}
R(U, \varphi V, U, \varphi V)= & R(U, \varphi V, V, \varphi U)+\varepsilon\{g(U, U) g(V, V) \\
& \left.-g(U, V)^{2}-g(U, \varphi V)^{2}\right\}, \\
R(U, \varphi U, V, \varphi U)= & R(U, \varphi U, U, \varphi V),
\end{aligned}
$$

for all $U, V \in \operatorname{Ker} \eta$. Putting $U+V$ in (4.4), and using(4.2), (4.5), (4.6) and the first Bianchi identity, we obtain

$$
\begin{aligned}
& 2 R(U, \varphi U, U, \varphi V)+2 R(V, \varphi V, V, \varphi U)+3 R(U, \varphi V, V, \varphi U)+R(U, V, U, V) \\
= & -c\left\{2 g(U, V)^{2}+2 g(U, U) g(U, V)+2 g(U, V) g(V, V)+g(U, U) g(V, V)\right\} .
\end{aligned}
$$

Replacing $V$ by $-V$ and then summing the resulting equation to the above equation gives

$$
3 R(U, \varphi V, V, \varphi U)+R(U, V, U, V)=-c\left\{2 g(U, V)^{2}+g(U, U) g(V, V)\right\} .
$$

Replacing $V$ by $\varphi V$ in (4.7) and then using the identities (4.2) and (4.5), we get

$$
4 R(U, V, U, V)=(c-3 \varepsilon)\left\{g(U, V)^{2}-g(U, U) g(V, V)\right\}-3(c+\varepsilon) g(U, \varphi V)^{2} .
$$

For $U, V, Z, W \in \operatorname{Ker} \eta$, we determine $R(U+Z, V+W, U+Z, V+W)$ and then using (4.8) we obtain

$$
4 R(U, V, Z, W)+4 R(U, W, Z, V)=(c-3 \varepsilon)\{g(U, V) g(Z, W)
$$




$$
\begin{gathered}
+g(U, W) g(V, Z)-2 g(U, Z) g(V, W)\}-3(c+\varepsilon)\{g(U, \varphi V) g(Z, \varphi W) \\
+g(U, \varphi W) g(Z, \varphi V)\} .
\end{gathered}
$$

Interchanging $V$ and $Z$ in (4.9), and then subtracting the resulting equation with (4.9) and by virtue of the first Bianchi identity we obtain

$$
\begin{aligned}
& 4 R(U, W, Z, V)=(c-3 \varepsilon)\{g(U, V) g(Z, W)-g(U, Z) g(V, W)\} \\
& -(c+\varepsilon)\{g(U, \varphi V) g(Z, \varphi W)-g(U, \varphi Z) g(V, \varphi W)+2 g(U, \varphi W) g(Z, \varphi V)\} .
\end{aligned}
$$

Now if $X, Y, Z, W \in T M$, then replacing $U, V, Z, W$ by $\varphi X, \varphi Y, \varphi Z, \varphi W$ in (4.10), and using (4.1), (4.2), and $\eta(R(X, Y) Z)=\eta(Y) g(X, Z)-\eta(X) g(Y, Z)$ we get (4.3). The converse is trivial.

Theorem 2. If a Kenmotsu pseudo-metric manifold has constant $\varphi$-sectional curvature $c$, then it is a space of constant curvature and $c=-\varepsilon$.

Proof. From (4.3), it is easy to obtain (3.7), where $a=\frac{1}{2}(n(c-3 \varepsilon)+(c+\varepsilon))$ and $b=\frac{-1}{2} \varepsilon(n+1)(c+\varepsilon)$. Since $a$ and $b$ are constants, from Corollary 3 it follows that $c=-\varepsilon$.

\section{SOME STRUCTURE THEOREMS}

The tangent space $T_{p} M$ of an almost contact pseudo-metric manifold $M$ can be decomposed as $T_{p} M=\varphi\left(T_{p} M\right) \oplus L\left(\xi_{p}\right)$, where $L\left(\xi_{p}\right)$ is the linear subspace of $T_{p} M$ generated by $\xi_{p}$. Thus the conformal curvature tensor $C$ is defined as a map

$$
C: T_{p} M \times T_{p} M \times T_{p} M \rightarrow \varphi\left(T_{p} M\right) \oplus L\left(\xi_{p}\right), \quad p \in M,
$$

such that

$$
\begin{aligned}
C(X, Y) Z= & R(X, Y) Z-\frac{1}{2 n-1}\{\operatorname{Ric}(Y, Z) X+g(Y, Z) Q X-R i c(X, Z) Y \\
& -g(X, Z) Q Y\}+\frac{r}{2 n(2 n-1)}\{g(Y, Z) X-g(X, Z) Y\} .
\end{aligned}
$$

Then there arise three cases:

- The projection of the image of $C$ in $\varphi\left(T_{p} M\right)$ is zero, that is,

$$
C(X, Y, Z, \varphi W)=0, \quad \text { for any } X, Y, Z, W \in T_{p} M .
$$

- Projection of the image of $C$ in $L\left(\xi_{p}\right)$ is zero, that is,

$$
C(X, Y) \xi=0, \quad \text { for all } X, Y \in T_{p} M .
$$

- Projection of the image of $\left.C\right|_{\varphi\left(T_{p} M\right) \times \varphi\left(T_{p} M\right) \times \varphi\left(T_{p} M\right)}$ in $\varphi\left(T_{p} M\right)$ is zero, that is,

$$
\varphi^{2} C(\varphi X, \varphi Y) \varphi Z=0, \quad \text { for all } X, Y, Z \in T_{p} M .
$$


An almost contact pseudo-metric manifold satisfying the cases (5.2), (5.3) and (5.4) are said to be conformally symmetric [14], $\xi$-conformally flat [13] and $\varphi$-conformally flat [2], respectively.

We begin with the following:

Theorem 3. Let $M$ be a $\xi$-conformally flat Kenmotsu pseudo-metric manifold of dimension higher than 3. Then the scalar curvature $r$ of $M$ satisfies

$$
D r=\varepsilon \xi(r) \xi,
$$

where D denotes gradient.

Proof. Since $M$ is $\xi$-conformally flat, from (5.3) the equation (5.1) becomes

$$
\begin{aligned}
R(U, V) \xi= & \frac{1}{2 n-1}\{\operatorname{Ric}(V, \xi) U+\varepsilon \eta(V) Q U-\operatorname{Ric}(U, \xi) V-\varepsilon \eta(U) Q V\} \\
& -\frac{\varepsilon r}{2 n(2 n-1)}\{\eta(V) U-\eta(U) V\},
\end{aligned}
$$

for any $U, V \in T M$, and this further gives

$$
\begin{aligned}
R(U, \xi) V= & \frac{1}{2 n-1}\{g(V, Q \xi) U+\varepsilon \eta(V) Q U-g(Q U, V) \xi-g(U, V) Q \xi\} \\
& -\frac{r}{2 n(2 n-1)}\{\varepsilon \eta(V) U-g(U, V) \xi\} .
\end{aligned}
$$

Putting $V=\xi$ in (5.6), then differentiating it covariently along $W$ and using (5.7), we get:

$$
\begin{aligned}
\left(\nabla_{W} R\right)(U, \xi) \xi= & \frac{1}{2 n-1}\left\{g\left(\left(\nabla_{W} Q\right) \xi, \xi\right) U+\varepsilon\left(\nabla_{W} Q\right) U-g\left(\left(\nabla_{W} Q\right) U, \xi\right) \xi\right. \\
& \left.-\varepsilon \eta(U)\left(\nabla_{W} Q\right) \xi\right\}-\frac{W r}{2 n(2 n-1)}\{\varepsilon U-\varepsilon \eta(U) \xi\} .
\end{aligned}
$$

Taking the inner product of the above equation with $Y$ and contracting with respect to $U$ and $W$ yield

$$
\begin{aligned}
\sum_{i=1}^{2 n+1} \varepsilon_{i} g\left(\left(\nabla_{e_{i}} R\right)\left(e_{i}, \xi\right) \xi, Y\right)= & \frac{1}{2 n-1}\left\{g\left(\left(\nabla_{Y} Q\right) \xi-\left(\nabla_{\xi} Q\right) Y, \xi\right)\right\} \\
& +\frac{\varepsilon(2 n-2)}{4 n(2 n-1)}\{Y r-\eta(Y) \xi(r)\},
\end{aligned}
$$

where $\left\{e_{i}\right\}$ is a pseudo-orthonormal basis in $M$ and $\varepsilon_{i}=g\left(e_{i}, e_{i}\right)$. From the second Bianchi identity we easily obtain

$$
\sum_{i=1}^{2 n+1} \varepsilon_{i} g\left(\left(\nabla_{e_{i}} R\right)(Y, \xi) \xi, e_{i}\right)=g\left(\left(\nabla_{Y} Q\right) \xi-\left(\nabla_{\xi} Q\right) Y, \xi\right) .
$$


Then from (5.8) and (5.9), noting that $n>1$ we get

$$
g\left(\left(\nabla_{Y} Q\right) \xi-\left(\nabla_{\xi} Q\right) Y, \xi\right)=\frac{\varepsilon}{4 n}\{Y r-\eta(Y) \xi(r)\} .
$$

Since $\nabla Q$ is symmetric, the above equation becomes

$$
g\left(\left(\nabla_{Y} Q\right) \xi, \xi\right)-g\left(\left(\nabla_{\xi} Q\right) \xi, Y\right)=\frac{\varepsilon}{4 n}\{Y r-\eta(Y) \xi(r)\} .
$$

From (3.4), the left hand side of above equation vanishes. Then (5.10) leads to $Y r=$ $\eta(Y) \xi(r)$ which gives (5.5).

Theorem 4. A Kenmotsu pseudo-metric manifold $M$ is $\xi$-conformally flat if and only if it is an $\eta$-Einstein manifold.

Proof. If $M$ is $\xi$-conformally flat, then

$$
\begin{aligned}
R(X, \xi) \xi= & \frac{1}{2 n-1}\{\operatorname{Ric}(\xi, \xi) X+\varepsilon Q X-\operatorname{Ric}(X, \xi) \xi-\varepsilon \eta(X) Q \xi\} \\
& -\frac{\varepsilon r}{2 n(2 n-1)}\{X-\eta(X) \xi\} .
\end{aligned}
$$

Making use of equations (3.1) and (3.4) in above gives

$$
Q=\left(\frac{r}{2 n}+\varepsilon\right) I-\left(\frac{\varepsilon r}{2 n}+2 n+1\right) \varepsilon \eta \otimes \xi
$$

which is equivalent to (3.10).

Conversely, suppose that $M$ is $\eta$-Einstein. Formula (5.1) gives

$$
\begin{aligned}
C(X, Y) \xi= & R(X, Y) \xi-\frac{1}{2 n-1}\{\operatorname{Ric}(Y, \xi) X+\varepsilon \eta(Y) Q X-\operatorname{Ric}(X, \xi) Y \\
& -\varepsilon \eta(X) Q Y\}+\frac{\varepsilon r}{2 n(2 n-1)}\{\eta(Y) X-\eta(X) Y\} .
\end{aligned}
$$

Now using identities (3.1), (3.4) and (3.13) results in

$$
\begin{aligned}
C(X, Y) \xi & =R(X, Y) \xi-\frac{1}{2 n-1}\left\{(2 n-\varepsilon a)+\frac{\varepsilon r}{2 n}\right\}(\eta(X) Y-\eta(Y) X) \\
& =R(X, Y) \xi-(\eta(X) Y-\eta(Y) X)=0,
\end{aligned}
$$

and this concludes the proof.

Theorem 5. A Kenmotsu pseudo-metric manifold of dimension higher than 3 is $\varphi$-conformally flat if and only if it is a space of constant cuvature $-\varepsilon$.

Proof. Note that the $\varphi$-conformally flat condition $\varphi^{2} C(\varphi X, \varphi Y) \varphi Z=0$ is equivalent to $C(\varphi X, \varphi Y, \varphi Z, \varphi W)=0$, and so from (5.1) we get

$$
\begin{aligned}
& R(\varphi X, \varphi Y, \varphi Z, \varphi W) \\
= & \frac{1}{2 n-1}\{\operatorname{Ric}(\varphi Y, \varphi Z) g(\varphi X, \varphi W)+g(\varphi Y, \varphi Z) \operatorname{Ric}(\varphi X, \varphi W) \\
& -\operatorname{Ric}(\varphi X, \varphi Z) g(\varphi Y, \varphi W)-g(\varphi X, \varphi Z) \operatorname{Ric}(\varphi Y, \varphi W)\}
\end{aligned}
$$




$$
-\frac{r}{2 n(2 n-1)}\{g(\varphi Y, \varphi Z) g(\varphi X, \varphi W)-g(\varphi X, \varphi Z) g(\varphi Y, \varphi W)\} .
$$

Let $\left\{E_{i}=e_{i}, E_{n+i}=\varphi e_{i}, E_{2 n+1}=\xi\right\}_{i=1}^{n}$ be a local pseudo-orthonormal $\varphi$-basis. Taking $X=W=E_{i}$ in (5.11) and summing, we get

$$
\begin{aligned}
\sum_{i=1}^{2 n} \varepsilon_{i} R & \left(\varphi E_{i}, \varphi Y, \varphi Z, \varphi E_{i}\right) \\
= & \sum_{i=1}^{2 n} \varepsilon_{i}\left[\frac { 1 } { 2 n - 1 } \left\{\operatorname{Ric}(\varphi Y, \varphi Z) g\left(\varphi E_{i}, \varphi E_{i}\right)+g(\varphi Y, \varphi Z) \operatorname{Ric}\left(\varphi E_{i}, \varphi E_{i}\right)\right.\right. \\
& \left.-\operatorname{Ric}\left(\varphi E_{i}, \varphi Z\right) g\left(\varphi Y, \varphi E_{i}\right)-g\left(\varphi E_{i}, \varphi Z\right) \operatorname{Ric}\left(\varphi Y, \varphi E_{i}\right)\right\} \\
& \left.-\frac{r}{2 n(2 n-1)}\left\{g(\varphi Y, \varphi Z) g\left(\varphi E_{i}, \varphi E_{i}\right)-g\left(\varphi E_{i}, \varphi Z\right) g\left(\varphi Y, \varphi E_{i}\right)\right\}\right] \\
= & \left(\frac{2 n-2}{2 n-1}\right) \operatorname{Ric}(\varphi Y, \varphi Z)+\frac{1}{2 n-1}\left(\frac{r}{2 n}+\varepsilon 2 n\right) g(\varphi Y, \varphi Z),
\end{aligned}
$$

where $\varepsilon_{i}=g\left(E_{i}, E_{i}\right)$. It can be easily verified that

$$
\begin{aligned}
\sum_{i=1}^{2 n} \varepsilon_{i} R\left(\varphi E_{i}, \varphi Y, \varphi Z, \varphi E_{i}\right) & =\operatorname{Ric}(\varphi Y, \varphi Z)-\varepsilon R(\xi, \varphi Y, \varphi Z, \xi) \\
& =\operatorname{Ric}(\varphi Y, \varphi Z)+\varepsilon g(\varphi Y, \varphi Z) .
\end{aligned}
$$

So that equation (5.12) becomes

$$
\operatorname{Ric}(\varphi Y, \varphi Z)=\left(\varepsilon+\frac{r}{2 n}\right) g(\varphi Y, \varphi Z) .
$$

Substituting this in (5.11), one obtains

$$
\begin{gathered}
R(\varphi X, \varphi Y, \varphi Z, \varphi W) \\
=\frac{r+4 n \varepsilon}{2 n(2 n-1)}\{g(\varphi Y, \varphi Z) g(\varphi X, \varphi W)-g(\varphi X, \varphi Z) g(\varphi Y, \varphi W)\} .
\end{gathered}
$$

From (4.2), (4.1), (3.2) and (2.2), we get

$$
\begin{aligned}
R(\varphi X, \varphi Y, \varphi Z, \varphi W)= & R(X, Y, Z, W)+\eta(Y) \eta(Z) g(X, W)-\eta(X) \eta(Z) g(Y, W) \\
& -\eta(Y) \eta(W) g(X, Z)+\eta(X) \eta(W) g(Y, Z) .
\end{aligned}
$$

Now (5.13) and (5.14) imply

$$
\begin{aligned}
R(X, Y, Z, W)= & \frac{r+4 n \varepsilon}{2 n(2 n-1)}\{g(\varphi Y, \varphi Z) g(\varphi X, \varphi W)-g(\varphi X, \varphi Z) g(\varphi Y, \varphi W)\} \\
& -\eta(Y) \eta(Z) g(X, W)+\eta(X) \eta(Z) g(Y, W) \\
& +\eta(Y) \eta(W) g(X, Z)-\eta(X) \eta(W) g(Y, Z) .
\end{aligned}
$$


Now taking the scalar product of (4.1) with $W$ and by virtue of (5.15) we get an equation and then contracting the resulting equation with respect to $X$ and $W$ gives

$$
(2 n-2)\left(\frac{r+4 n \varepsilon}{2 n(2 n-1)}+\varepsilon\right) g(Y, \varphi Z)=0 .
$$

Since $n>1$, it follows that

$$
r=-\varepsilon 2 n(2 n+1) .
$$

Using (5.16) and (2.2) in (5.15), we get

$$
R(X, Y, Z, W)=-\varepsilon\{g(Y, Z) g(X, W)-g(X, Z) g(Y, W)\},
$$

and so that the manifold is of constant curvature $-\varepsilon$.

The converse is trivial.

Corollary 4. A conformally flat Kenmotsu pseudo-metric manifold of dimension higher than 3 is a space of constant cuvature $-\varepsilon$.

The above corollary for Riemannian case has been proved in [9].

Now contracting (5.15), we obtain (3.10). Thus we can state the following:

Corollary 5. A $\varphi$-conformally flat Kenmotsu pseudo-metric manifold is an $\eta$ Einstein manifold.

In view of Theorem 4 and Corrollary 5, we have the following:

Corollary 6. A $\varphi$-conformally flat Kenmotsu pseudo-metric manifold is always $\xi$-conformally flat.

\section{RICCI SOLITON ON KENMOTSU PSEUDO-METRIC MANIFOLDS}

A Ricci soliton on a pseudo-Riemannian manifold $(M, g)$ is defined by

$$
\left(£_{V} g\right)(X, Y)+2 \operatorname{Ric}(X, Y)+2 \lambda g(X, Y)=0,
$$

where $\lambda$ is a constant. Ricci soliton is a natural generalization of the Einstein metric (that is, $\operatorname{Ric}(X, Y)=\operatorname{ag}(X, Y)$, for some constant $a$ ), and is a special self similar solution of Hamilton's Ricci flow (see [8]) $\frac{\partial}{\partial t} g(t)=-2 R i c(t)$ with initial condition $g(0)=g$. We say that the Ricci soliton is steady when $\lambda=0$, expanding when $\lambda>0$ and shrinking when $\lambda<0$.

Before producing the main results, we prove the following:

Lemma 2. A Kenmotsu pseudo-metric manifold $(M, g)$ satisfies

$$
\begin{gathered}
\left(\nabla_{X} Q\right) \xi=-Q X-2 n \varepsilon X, \\
\left(\nabla_{\xi} Q\right) X=-2 Q X-4 n \varepsilon X .
\end{gathered}
$$


Proof. Differentiating $Q \xi=-2 n \varepsilon \xi$, and recalling (2.6) provide (6.2).

Now differentiating (3.1) along $W$ leads to

$$
\left(\nabla_{W} R\right)(X, Y) \xi=-R(X, Y) W+\varepsilon g(X, W) Y-\varepsilon g(Y, W) X .
$$

Contracting this with respect to $X$ and $W$ gives us

$$
\sum_{i=1}^{2 n+1} \varepsilon_{i} g\left(\left(\nabla_{e_{i}} R\right)\left(e_{i}, Y\right) \xi, Z\right)=\operatorname{Ric}(Y, Z)+2 n g(Y, Z) .
$$

From the second Bianchi identity, one can easily obtain

$$
\sum_{i=1}^{2 n+1} \varepsilon_{i} g\left(\left(\nabla_{e_{i}} R\right)(Z, \xi) Y, e_{i}\right)=g\left(\left(\nabla_{Z} Q\right) \xi, Y\right)-g\left(\left(\nabla_{\xi} Q\right) Z, Y\right) .
$$

Fetching (6.5) into (6.4) and with the aid of (6.2), we infer that

$$
g\left(\left(\nabla_{\xi} Q\right) Z, Y\right)=-2 \operatorname{Ric}(Y, Z)-4 n g(Y, Z),
$$

which proves (6.3).

Theorem 6. Let $(M, g)$ be a Kenmotsu pseudo-metric manifold. If $(g, V)$ is a Ricci soliton, then the soliton constant $\lambda=2 n \varepsilon$, and so the soliton is either expanding or shrinking depending on the casual character of the Reeb vector field $\xi$.

Proof. Differentiating (6.1) covariantly along $Z$ gives

$$
\left(\nabla_{Z} £_{V} g\right)(X, Y)=-2\left(\nabla_{Z} R i c\right)(X, Y) .
$$

From Yano [12], we know the following well known commutation formula:

$$
\begin{aligned}
\left(£_{V} \nabla_{X} g-\nabla_{X} £_{V} g\right. & \left.-\nabla_{[V, X]} g\right)(Y, Z) \\
& =-g\left(\left(£_{V} \nabla\right)(X, Y), Z\right)-g\left(\left(£_{V} \nabla\right)(X, Z), Y\right),
\end{aligned}
$$

for all $X, Y, Z \in T M$. Since $\nabla g=0$, the previous equation gives

$$
\left(\nabla_{X} £_{V} g\right)(Y, Z)=g\left(\left(£_{V} \nabla\right)(X, Y), Z\right)+g\left(\left(£_{V} \nabla\right)(X, Z), Y\right),
$$

for all $X, Y, Z \in T M$. As $£_{V} \nabla$ is a symmetric, it follows from (6.7) that

$$
\begin{aligned}
& g\left(\left(£_{V} \nabla\right)(X, Y), Z\right) \\
& =\frac{1}{2}\left(\nabla_{X} £_{V} g\right)(Y, Z)+\frac{1}{2}\left(\nabla_{Y} £_{V} g\right)(Z, X)-\frac{1}{2}\left(\nabla_{Z} £_{V} g\right)(X, Y) .
\end{aligned}
$$

Making use of (6.6) in (6.8) we have

$$
g\left(\left(£_{V} \nabla\right)(X, Y), Z\right)=\left(\nabla_{Z} R i c\right)(X, Y)-\left(\nabla_{X} R i c\right)(Y, Z)-\left(\nabla_{Y} R i c\right)(Z, X) .
$$

Putting $Y=\xi$ in (6.9) and using (6.2) and (6.3), we obtain

$$
\left(£_{V} \nabla\right)(X, \xi)=2 Q X+4 n \varepsilon X .
$$


Differentiating the preceding equation along $Y$ and using (2.6), we obtain

$$
\left(\nabla_{Y} £_{V} \nabla\right)(X, \xi)=-\left(£_{V} \nabla\right)(X, Y)+2 \eta(Y)\{Q X+2 n \varepsilon X\}+2\left(\nabla_{Y} Q\right) X .
$$

Feeding the above obtained expression into the following relation (see [12])

$$
\left(£_{V} R\right)(X, Y) Z=\left(\nabla_{X} £_{V} \nabla\right)(Y, Z)-\left(\nabla_{Y} £_{V} \nabla\right)(X, Z),
$$

and using the symmetry of $£_{V} \nabla$, we immediately obtain

$$
\begin{aligned}
\left(£_{V} R\right)(X, Y) \xi= & 2 \eta(X)\{Q Y+2 n \varepsilon Y\}-2 \eta(Y)\{Q X+2 n \varepsilon X\} \\
& +2\left\{\left(\nabla_{X} Q\right) Y-\left(\nabla_{Y} Q\right) X\right\} .
\end{aligned}
$$

Setting $Y=\xi$ in the foregoing equation, we get

$$
\left(£_{V} R\right)(X, \xi) \xi=0 .
$$

Now taking the Lie-derivative of $R(X, \xi) \xi=-X+\eta(X) \xi$ along $V$ gives

$$
\left(£_{V} R\right)(X, \xi) \xi-2 \eta\left(£_{V} \xi\right) X+\varepsilon g\left(X, £_{V} \xi\right) \xi=\left(£_{V} \eta\right)(X) \xi
$$

which by virtue of (6.12) becomes

$$
\left(£_{V} \eta\right)(X) \xi=-2 \eta\left(£_{V} \xi\right) X+\varepsilon g\left(X, £_{V} \xi\right) \xi .
$$

With the help of (3.4), the equation (6.1) takes the form

$$
\left(£_{V} g\right)(X, \xi)=-2 \lambda \varepsilon \eta(X)+4 n \eta(X) .
$$

Changing $X$ to $\xi$ in the aforementioned equation gives

$$
\eta\left(£_{V} \xi\right)=\lambda-2 n \varepsilon
$$

Now Lie-differentiating $\eta(X)=\varepsilon g(X, \xi)$ yields $\left(£_{V} \eta\right)(X)=\varepsilon\left(£_{V} g\right)(X, \xi)+\varepsilon g\left(X, £_{V} \xi\right)$. Using this and (6.15) in (6.13) provides $(\lambda-2 n \varepsilon)(X-\eta(X) \xi)=0$. Tracing the previous equation yield $\lambda=2 n \varepsilon$.

Corollary 7. A Kenmotsu manifold admitting the Ricci soliton is always expanding with $\lambda=2 n$.

Lemma 3. Let $(M, g)$ be a Kenmotsu pseudo-metric manifold. If $(g, V)$ is a Ricci soliton, then the Ricci tensor satisfies

$$
\left(£_{V} R i c\right)(X, \xi)=-X(r)+\xi(r) \eta(X) .
$$

Proof. Contracting equation (6.11) with respect to $X$ and recalling the well-known formulas

we easily obtain

$$
\operatorname{div} Q=\frac{1}{2} D r \quad \text { and } \quad \operatorname{trace} \nabla Q=D r
$$

$$
\left(£_{V} \operatorname{Ric}\right)(Y, \xi)=-Y(r)-2 \eta(Y)\{r+\varepsilon 2 n(2 n+1)\} .
$$


Substituting $Y=\xi$, we have $\left(£_{V} R i c\right)(\xi, \xi)=-\xi(r)-2\{r+\varepsilon 2 n(2 n+1)\}$. On the other hand, contracting (6.12) gives $\left(£_{V} R i c\right)(\xi, \xi)=0$. Using this in the previous equation leads to

$$
\xi(r)=-2(r+\varepsilon 2 n(2 n+1)) .
$$

Hence (6.18) and (6.17) give (6.16).

Combining Theorem 3 and 4, we state the following:

Lemma 4. An $\eta$-Einstein Kenmotsu pseudo-metric manifold $M$ of dimension higher than 3 satisfies

$$
D r=\varepsilon \xi(r) \xi
$$

Now we prove:

Theorem 7. Let $(M, g)$ be an $\eta$-Einstein Kenmotsu pseudo-metric manifold of dimension higher than 3. If $(g, V)$ is a Ricci soliton, then $M$ is Einstein.

Proof. Making use of (6.19) in (6.16), we have $\left(£_{V} R i c\right)(X, \xi)=0$. Now, Liedifferentiating the first relation of (3.4) along $V$, using (3.10), (6.14), $\lambda=2 n \varepsilon$ and $\eta\left(£_{V} \xi\right)=0$, we obtain

$$
(r+\varepsilon 2 n(2 n+1)) £_{V} \xi=0 .
$$

If $r=-\varepsilon 2 n(2 n+1)$, then (3.10) shows that $M$ is Einstein.

So we assume $r \neq-\varepsilon 2 n(2 n+1)$ in some open set $\mathcal{O}$ of $M$. Hence $£_{V} \xi=0$ on $\mathcal{O}$, and so it follows from (2.6) that

$$
\nabla_{\xi} V=V-\eta(V) \xi
$$

Clearly, (6.14) shows that $\left(£_{V} g\right)(X, \xi)=0$. This together with (6.20), we have

$$
g\left(\nabla_{X} V, \xi\right)=-g\left(\nabla_{\xi} V, X\right)=-g(X, V)+\eta(X) \eta(V) .
$$

From Duggal and Sharma [5], we know that

$$
\left(£_{V} \nabla\right)(X, Y)=\nabla_{X} \nabla_{Y}-\nabla_{\nabla_{X} Y} V+R(V, X) Y .
$$

Setting $Y=\xi$ in the above equation and by virtue of (2.6), (3.1), (6.20) and (6.21), we have $r=-\varepsilon 2 n(2 n+1)$. This leads to a contradiction as $r \neq-\varepsilon 2 n(2 n+1)$ on $\mathcal{O}$ and completes the proof.

Now we consider Kenmotsu pseudo-metric 3-manifolds which admits Ricci solitons.

Theorem 8. Let $(M, g)$ be a Kenmotsu pseudo-metric 3-manifold. If $(g, V)$ is a Ricci soliton, then $M$ is of constant curvature $-\varepsilon$. by

Proof. The Riemannian curvature tenor of pseudo-Riemannian 3-manifold is given

$$
\begin{aligned}
R(X, Y) Z= & g(Y, Z) Q X-g(X, Z) Q Y+g(Q Y, Z) X-g(Q X, Z) Y \\
& -\frac{r}{2}\{g(Y, Z) X-g(X, Z) Y\} .
\end{aligned}
$$


Taking $Y=Z=\xi$ in (6.22) and using (3.1) and (3.4) gives

$$
Q=\left(\frac{r}{2}+1\right) I-\left(\frac{r}{2}+3\right) \eta \otimes \xi .
$$

Making use of this in (6.11) gives

$$
\begin{aligned}
\left(£_{V} R\right)(X, Y) \xi= & X(r)\{Y-\eta(Y) \xi\}+Y(r)\{-X+\eta(X) \xi\} \\
& -(r+6 \varepsilon)\{\eta(Y) X-\eta(X) Y\} .
\end{aligned}
$$

Replacing $Y$ by $\xi$ in the above equation and comparing it with (6.12), we obtain

$$
\{\xi(r)+(r+6 \varepsilon)\}\{-X+\eta(X) \xi\}=0 .
$$

Contracting the above equation gives $\xi(r)+(r+6 \varepsilon)=0$, and consequently it follows from (6.18) that $r=-6 \varepsilon$. Then from (6.23) we have $Q X=-2 \varepsilon X$, and substituting this into (6.22) shows that $M$ is of constant curvature $-\varepsilon$.

Corollary 8. There does not exist a Kenmotsu pseudo-metric manifold $(M, g)$ admitting the Ricci soliton $(g, V=\xi)$.

Proof. If $V=\xi$, then from (2.8) the Ricci soliton equation (6.1) would become

$$
R i c=-(1+\lambda) g+\varepsilon \eta \otimes \eta,
$$

which means $M$ is $\eta$-Einstein. Then due to Theorem 7 and $8, M$ must be Einstein, and this will be a contradiction to equation (6.25) as $\varepsilon \neq 0$.

Remark 1. Clearly, Theorem 7 and 8 are generalizations of the results of Ghosh proved in [6] and [7]. Note that our approach and technique to obtain the result is different to that of Ghosh.

Now we provide an example of a Kenmotsu pseudo-metric 3-manifold which admits a Ricci soliton and verify our results.

Example 1. Let $M=N \times I$, where $N$ is an open connected subset of $\mathbb{R}^{2}$ and $I$ is an open interval in $\mathbb{R}$. Let $(x, y, z)$ be the Cartesian coordinates in $M$. Define the structure $(\varphi, \xi, \eta, g)$ on $M$ as follows:

$$
\begin{aligned}
\varphi\left(\frac{\partial}{\partial x}\right) & =\frac{\partial}{\partial y}, \quad \varphi\left(\frac{\partial}{\partial y}\right)=-\frac{\partial}{\partial x}, \quad \varphi\left(\frac{\partial}{\partial z}\right)=0, \\
\xi & =\frac{\partial}{\partial z}, \quad \eta=d z \\
\left(g_{i j}\right) & =\left(\begin{array}{ccc}
e^{2 z} & 0 & 0 \\
0 & e^{2 z} & 0 \\
0 & 0 & \varepsilon
\end{array}\right) .
\end{aligned}
$$


Now from Koszul's formula, the Levi-Civita connection $\nabla$ is given by

$$
\begin{array}{lll}
\nabla_{\partial_{1}} \partial_{1}=-\varepsilon e^{2 z} \partial_{3}, & \nabla_{\partial_{1}} \partial_{2}=0, & \nabla_{\partial_{1}} \partial_{3}=\partial_{1}, \\
\nabla_{\partial_{2}} \partial_{1}=0, & \nabla_{\partial_{2}} \partial_{2}=-\varepsilon e^{2 z} \partial_{3}, & \nabla_{\partial_{2}} \partial_{3}=\partial_{2}, \\
\nabla_{\partial_{3}} \partial_{1}=\partial_{1}, & \nabla_{\partial_{3}} \partial_{2}=\partial_{2}, & \nabla_{\partial_{3}} \partial_{3}=0,
\end{array}
$$

where $\partial_{1}=\frac{\partial}{\partial x}, \partial_{2}=\frac{\partial}{\partial y}$ and $\partial_{3}=\frac{\partial}{\partial z}$. From (6.26), one can easily verify

$$
\left(\nabla_{\partial_{i}} \varphi\right) \partial_{j}=-\eta\left(\partial_{j}\right) \varphi \partial_{i}-\varepsilon g\left(\partial_{i}, \varphi \partial_{j}\right) \xi,
$$

for all $i, j=1,2,3$, and so $M$ is a Kenmotsu pseudo-metric manifold with the above $(\varphi, \xi, \eta, g)$ structure.

With the help of (6.26), we find that:

$$
\begin{gathered}
R\left(\partial_{1}, \partial_{2}\right) \partial_{3}=R\left(\partial_{2}, \partial_{3}\right) \partial_{1}=R\left(\partial_{1}, \partial_{3}\right) \partial_{2}=0, \\
R\left(\partial_{1}, \partial_{3}\right) \partial_{1}=R\left(\partial_{2}, \partial_{3}\right) \partial_{2}=\varepsilon e^{2 z} \partial_{3}, \\
R\left(\partial_{1}, \partial_{2}\right) \partial_{1}=\varepsilon e^{2 z} \partial_{2}, \quad R\left(\partial_{2}, \partial_{3}\right) \partial_{3}=-\partial_{2}, \\
R\left(\partial_{1}, \partial_{3}\right) \partial_{3}=-\partial_{1}, \quad R\left(\partial_{1}, \partial_{2}\right) \partial_{2}=-\varepsilon e^{2 z} \partial_{1} .
\end{gathered}
$$

Let $e_{1}=e^{-z} \partial_{1}, e_{2}=e^{-z} \partial_{2}$ and $e_{3}=\xi=\partial_{3}$. Clearly, $\left\{e_{1}, e_{2}, e_{3}\right\}$ forms an orthonormal $\varphi$-basis of vector fields on $M$. Making use of (6.28) one can easily show that $M$ is Einstein, that is, $\operatorname{Ric}(Y, Z)=-2 \varepsilon g(Y, Z)$, for any $Y, Z \in T M$.

Let us consider the vector field

$$
V=\alpha \frac{\partial}{\partial y}
$$

where $\alpha$ is a non-zero constant. Making use of (6.26) one can easily show that $V$ is Killing with respect to $g$, that is, we have

$$
\left(£_{V} g\right)(X, Y)=g\left(\nabla_{X} V, Y\right)+g\left(\nabla_{Y} V, X\right)=0,
$$

for any $X, Y \in T M$. Hence $g$ is a Ricci soliton, that is, (6.1) holds true with $V$ as in (6.29) and $\lambda=2 \varepsilon$. Further (6.28) shows that

$$
R(X, Y) Z=-\varepsilon\{g(Y, Z) X-g(X, Z) Y\},
$$

for any $X, Y \in T M$, which means $M$ is of constant curvature $-\varepsilon$ and so Theorem 8 is verified.

\section{ACKNOWLEDGEMENT}

The authors would like to thank the reviewer for careful and thorough reading of this manuscript and thankful for helpful suggestions towards the improvement of this paper. 


\section{REFERENCES}

[1] A. Bejancu and K. L. Duggal, "Real hypersurfaces of indefinite Kaehler manifolds." Int. J. Math. Math. Sci., vol. 16, no. 3, pp. 545-556, 1993, doi: 10.1155/S0161171293000675.

[2] J. Cabrerizo, L. Fernandez, M. Fernandez, and G. Zhen, "The strucure of a class of $K$-contact manifolds." Acta Math. Hungar., vol. 82, no. 4, pp. 331-340, 1990, doi: 10.1023/A:1006696410826.

[3] G. Calvaruso and D. Perrone, "Contact pseudo-metric manifolds." Differ. Geom. Appl., vol. 28, no. 2, pp. 615-634, 2010, doi: 10.1016/j.difgeo.2010.05.006.

[4] K. L. Duggal, "Space time manifolds and contact structures." Int. J. Math. Math. Sci., vol. 13, no. 3, pp. 545-554, 1990, doi: 10.1155/S0161171290000783.

[5] K. L. Duggal and R. Sharma, Symmetries of Spacetimes and Riemannian Manifolds. Kluwer: Springer, 1999. doi: 10.1007/978-1-4615-5315-1.

[6] A. Ghosh, "Kenmotsu 3-metric as a Ricci soliton." Chaos Solitons Fractals, vol. 44, pp. 647-650, 2011.

[7] A. Ghosh, "An $\eta$-Einstein Kenmotsu metric as a Ricci soliton," Publ. Math. Debrecen, vol. 82, pp. 591-598, 2013.

[8] R. Hamilton, "The Ricci flow on surfaces." Contemp. Math., vol. 71, pp. 237-261, 1988.

[9] K. Kenmotsu, "A class of almost contact Riemannian manifolds.” Tohoku Math. J., vol. 24, no. 1, pp. 93-103, 1972, doi: 10.2748/tmj/1178241594.

[10] T. Takahashi, "Sasakian manifold with pseudo-Riemannian metrics." Tohoku Math. J., vol. 21, no. 2, pp. 271-290, 1969, doi: 10.2748/tmj/1178242996.

[11] Y. Wang and X. Liu, "Almost Kenmotsu pseudo-metric manifolds." An. Stiint. Univ. Al. I. Cuza Iasi. Mat. (N.S.), vol. LXII, no. 1, pp. 241-256, 2016.

[12] K. Yano, Integral formulas in Riemannian geometry. New York: Marcel Dekker, 1970.

[13] G. Zhen, J. Cabrerizo, L. Fernandez, and M. Fernandez, "On $\xi$-conformally flat contact metric manifolds." Indian J. Pure Appl. Math., vol. 28, pp. 725-734, 1997.

[14] G. Zhen, "Conformally symmetric K-contact manifolds." Chinese Quart. J. Math., vol. 7, no. 1, pp. 5-10, 1992.

Authors' addresses

Devaraja Mallesha Naik

Department of Mathematics, Kuvempu University, Shankaraghatta, 577-451 Shivamogga, India

E-mail address: devarajamaths@gmail.com

\section{Venkatesha}

Kuvempu University, Department of Mathematics, Shankaraghatta, 577-451 Shivamogga, India

E-mail address: vensmath@gmail.com

\section{D.G. Prakasha}

Department of Mathematics, Karnatak University, Dharwad, Karnataka, India and Department of Mathematics, Davangere University, Davangere, India

E-mail address: prakashadg@gmail.com 